\title{
Neumotórax espontáneo primario idiopático en adolescente
}

\author{
Idiopathic primary spontaneous pneumothorax in an adolescent
}

\author{
Alicia Martínez-Sebastián* \\ * Hospital Universitario Doctor Peset. Valencia, España.
}

\section{RESUMEN}

Introducción: El neumotórax espontáneo del niño es una condición rara y, en ocasiones, el diagnóstico es difícil. Es más frecuente en pacientes del sexo masculino, adolescentes, con fenotipo leptosómico y fumadores. Caso clínico: Varón de 16 años que presentó neumotórax espontáneo tras realizar ejercicio, que se complicó con hemotórax por iatrogenia, así como con colapso del pulmón ipsilateral. Posterior a tratamiento específico, presentó buena evolución clínica. Conclusiones: El pediatra debe estar entrenado en la detección del neumotórax de manera oportuna para evitar posibles complicaciones.

Palabras clave: Neumotórax espontáneo, hemotórax, drenaje, adolescente.

\section{INTRODUCCIÓN}

El neumotórax del niño es poco frecuente y se define por la presencia de aire en el espacio pleural, que en el caso del neumotórax espontáneo se situaría entre la pleura visceral y el pulmón. La irrupción de aire en la pleura puede ser secundaria a la ruptura de blebs o de bulas que se localizan, casi siempre, en los vértices pulmonares donde el gradiente de presión pleural es más elevado que en la base y, por lo tanto, las rupturas alveolares son más frecuentes. ${ }^{1}$ Éstas pueden verse favorecidas por situaciones que producen gradientes de presión importante como

\begin{abstract}
Introduction: Spontaneous pneumothorax in children is a rare condition and sometimes the diagnosis is difficult. It is more frequent in male patients, adolescents, with the leptosomal phenotype and smokers. Clinical case: A 16-year-old man presented a spontaneous pneumothorax after exercising, which was complicated, with iatrogenic hemothorax, as well as ipsilateral lung collapse. After specific treatment, he presented good clinical evolution. Conclusions: Pediatricians must be trained in the detection of pneumothorax in a timely manner to avoid possible complications.
\end{abstract}

Keywords: Spontaneous pneumothorax, hemothorax, drainage, adolescent.

los esfuerzos físicos, incluyendo el levantamiento de pesas.

Se observan dos picos de frecuencia en la aparición de un neumotórax espontáneo. El primero ocurre entre los 15-34 años, mientras que el segundo aparece después de los 55 años. La incidencia del neumotórax espontáneo primario es inexacta; sin embargo, predomina en el sexo masculino, oscilando de 18-28/100,000 casos al año en hombres y 1.2-6/100,000 en mujeres. ${ }^{2}$

Presentamos el caso de un adolescente con neumotórax espontáneo primario idiopático tras realizar ejercicio, en quien por iatrogenia, además, tuvo hemotórax iatrógeno y colapso del pulmón ipsilateral tras

Correspondencia: Alicia Martínez-Sebastián, aliciamarsebastian@gmail.com

Citar como: Martínez-Sebastián A. Neumotórax espontáneo primario idiopático en adolescente. Rev Mex Pediatr. 2021; 88(1): 22-25. https:// dx.doi.org/10.35366/99415 
realizar punción para drenaje terapéutico. Con este caso queremos destacar la importancia de realizar un manejo adecuado, así como repasar los aspectos más importantes de esta patología y sus posibles complicaciones.

\section{PRESENTACIÓN DEL CASO}

Adolescente varón de 16 años que acude a urgencias por dolor en hemitórax izquierdo de 10 horas de evolución, sin dificultad respiratoria ni fiebre. Como único antecedente refiere haber realizado ejercicio con levantamiento de pesas el día previo. El paciente pesa $63 \mathrm{~kg}$ y mide $187 \mathrm{~cm}$ (IMC 18.02), no fumador.

A la exploración física (EF) se encuentra con constantes vitales dentro de la normalidad, con buena saturación. Se destaca hipofonesis en región anterior de ápex pulmonar izquierdo, por lo que se realiza una radiografía de tórax en la que se aprecia un neumotórax izquierdo del 15\% (Figura 1) según el índice de Light. El electrocardiograma y exámenes de laboratorio fueron normales.

Seis horas después, refiere dolor intenso a pesar de analgesia, por lo que se toma nueva radiografía, observando aumento del neumotórax al 20\% (Figura 2). Se decide colocación de drenaje torácico en segundo espacio intercostal izquierdo con posterior aspiración

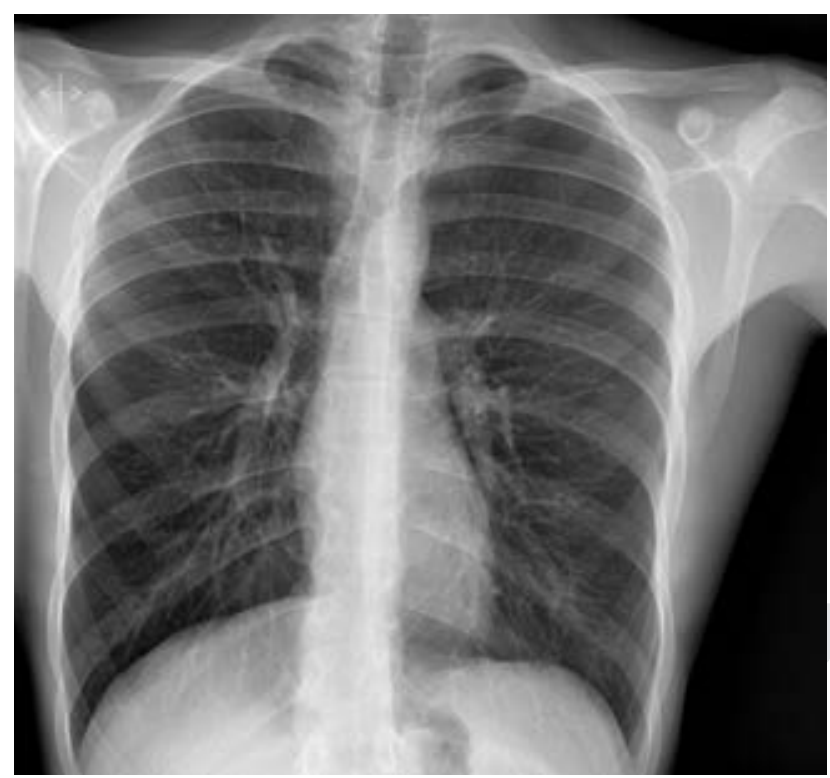

Figura 1: Rx tórax postero-anterior en bipedestación: neumotórax pequeño izquierdo de 15\% según el índice de Light, que no condiciona desplazamiento mediastínico.

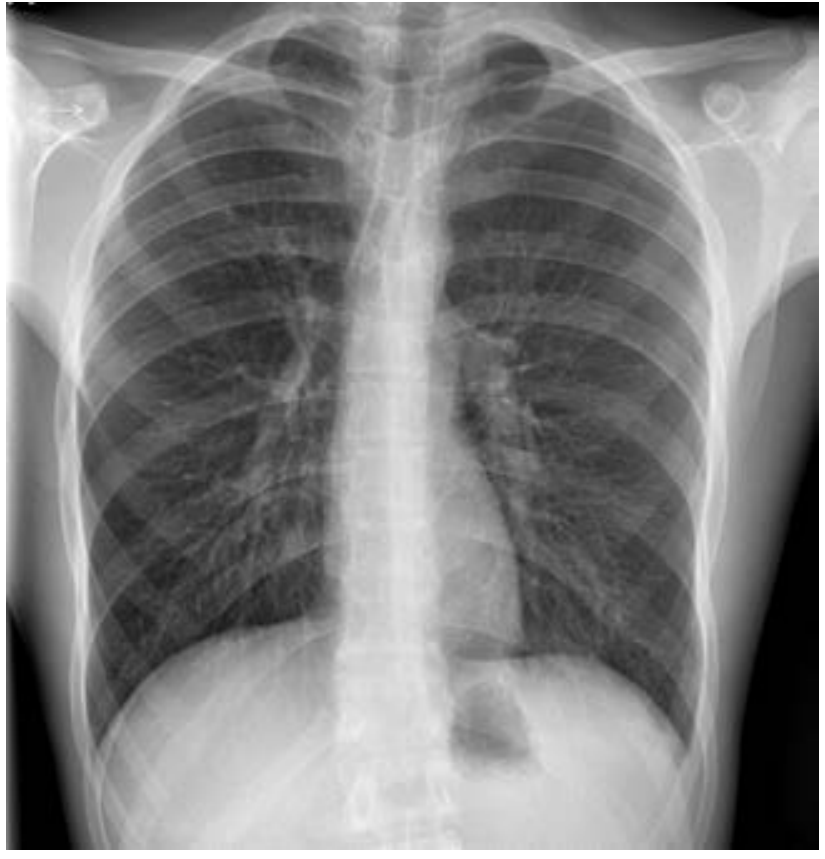

Figura 2: Rx tórax postero-anterior en bipedestación: discreto aumento del neumotórax izquierdo del 20\% (moderado) según el índice de Light.

con Pleur-evac ${ }^{\circledR}$. El control radiológico a las 12 horas se observa con mejoría, por lo que se cierra aspiración. Sin embargo, a las 18 horas el paciente presenta empeoramiento del dolor torácico de características pleuríticas, a lo que se agregó drenaje de líquido hemático de aproximadamente $300 \mathrm{~mL}$, sospechando de hemotórax. El hematocrito fue de 48\%.

En tomografía axial vascular se evidencia sangrado activo, con probable dependencia de primera arteria intercostal izquierda. Mediante radiología intervencionista se realiza embolización con coil de $3 \mathrm{~mm}$, aspirando $350 \mathrm{~mL}$ de líquido hemático, y posteriormente desaparece el derrame. Se envía a UCI para monitorización. A las 48 h, presenta empeoramiento clínico y radiológico, ya que aumentó el neumotórax al 65\%, se detecta colapso pulmonar ipsilateral (Figura 3) y derrame pleural. Se decide realizar aspiración con jeringa a través de Pleurocath $^{\circledR}$ obteniendo $70 \mathrm{~mL}$ drenaje hemático con componente aéreo. Se deja aspiración a $30 \mathrm{cmH}_{2} \mathrm{O}$, la cual se retiró tras la reexpansión pulmonar. Cabe señalar que permaneció hemodinámicamente estable en todo momento, sin necesidad de soporte hemodinámico ni transfusional. El paciente se egresó sin más eventualidades. 


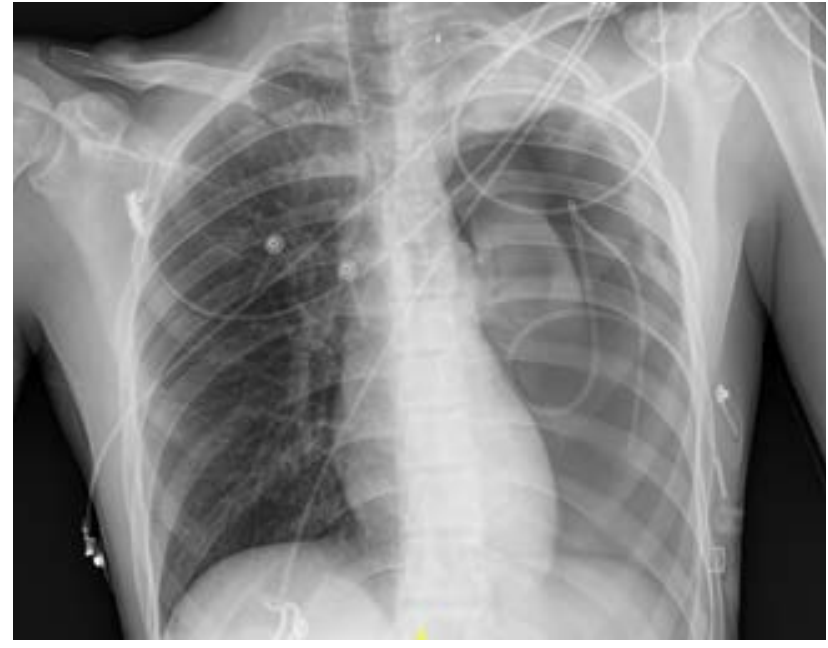

Figura 3: Rx tórax portátil en decúbito: colapso pulmonar izquierdo por neumotórax en estudio actual del $65 \%$ según el índice de Light, con desplazamiento mediastínico hacia hemitórax derecho.

\section{DISCUSIÓN}

El neumotórax espontáneo habitualmente se resuelve de forma satisfactoria tras su drenaje mediante punción-aspiración, válvula de Heimlich/ minidrenaje o tubo, estimándose que las recidivas pueden ocurrir en alrededor del $30 \% .^{2}$ El caso que presentamos representa a los pacientes que no llegan a una fácil resolución, ya que hubo múltiples complicaciones.

El neumotórax se considera espontáneo si se produce en ausencia de enfermedad pulmonar subyacente. Éstos se clasifican en primarios o secundarios, que a su vez se dividen en iatrogénicos y traumáticos. En cuanto a su etiopatogenia, la estatura elevada asociada al sexo masculino, así como el tabaquismo activo son factores de riesgo reconocidos. La progresión de los blebs o las bulas, en los individuos longilíneos y de bajo índice de masa corporal, se debe al incremento de la presión mecánica, resultado de la combinación de una forma elipsoide aplanada del vértice pulmonar y de la presión sobre el pulmón, causada por unas primeras costillas prominentes. ${ }^{3}$

El cuadro clínico generalmente consiste en un cuadro agudo de dolor torácico y disnea. Mientras que los datos habituales en la EF incluyen disminución de movilidad en el lado afectado, timpanismo a la percusión y disminución del murmullo vesicular; sin embargo, en la edad pediátrica estos datos no siempre se reconocen. El diagnóstico de certeza se basa en la radiografía de tórax postero-anterior y lateral, en bipedestación y en inspiración, en la cual se confirma el diagnóstico, al mostrar la línea pleural visceral, que debe buscarse en el vértice y la zona axilar, con ausencia de trama vascular periférica. En las formas completas, el pulmón está totalmente retraído en el hilio pulmonar.

En cuanto a su clasificación, debemos mencionar que la diversidad de métodos publicados para cuantificar el tamaño del neumotórax refleja la falta de consenso sobre el sistema a emplear, pues la conformación inconstante y poco uniforme del parénquima pulmonar durante el episodio suele conducir a subestimar su volumen. Por lo general, la estimación del tamaño del neumotórax se basa en cálculos realizados en la placa torácica en bipedestación, como el índice de Light (el más utilizado en Europa, basado en el diámetro al cubo del radio del pulmón y el hemitórax [\% neumotórax $=100$ (1-diámetro pulmón) /diámetro del hemitórax]) o las puntuaciones de Collins o de Rhea. ${ }^{4,5}$

La cuantificación del despegamiento es muy controvertida. En los EEUU se recomienda evaluar el neumotórax por la distancia entre el vértice del tórax y la cúpula pulmonar, considerando que es importante $>3 \mathrm{~cm}$. Mientras que en Reino Unido se toma en cuenta la distancia entre la pared torácica y el borde externo del pulmón, en la zona del hilio; así, un neumotórax es grande cuando $>2 \mathrm{~cm}$. Por su parte, la Sociedad Española de Neumología y Cirugía torácica clasifica al neumotórax en parcial (separación de la pleura visceral en parte de la cavidad pleural), completo (separación en toda la cavidad) o total (muñón pulmonar). ${ }^{5}$

El tratamiento, por lo general, varía en función del tamaño del neumotórax y la condición del paciente. De acuerdo con la Sociedad Española de Cirugía torácica (SECT), en su guía publicada en el 2018, para los casos de neumotórax pequeños $(<20 \%$ según el índice de Light) o parciales, en sujetos estables, primero se recomienda la observación (ya que el aire se reabsorbe alrededor del 1 al 2\% de su volumen al día), en ocasiones con oxigenoterapia (para corregir una posible hipoxemia y asegurar una reabsorción cuatro veces más rápida). Cuando los pacientes tienen deterioro clínico o radiológico, como en nuestro caso, se tratará como un neumotórax completo/total o inestable, mediante la punción con aguja, asociada o no al drenaje torácico. ${ }^{5}$ Mientras que la cirugía torácica, por toracoscopía, está indicada en los neumotórax persistentes a pesar 
del drenaje torácico, y probablemente para las formas recidivantes. ${ }^{6}$

En el caso que presentamos se siguieron las recomendaciones descritas, pero al colocar drenaje se produjo lesión de la arteria intercostal izquierda y aparición de hemotórax (presencia de sangre en el espacio pleural). Cuando existe este tipo de complicación, se debe tener en cuenta que puede ser grave, por lo que un diagnóstico precoz del mismo resulta imprescindible. El síntoma principal es el dolor de tipo pleurítico localizado en el hemitórax afectado; el dolor se puede confundir por el condicionado al colocar drenaje o por la movilización del enfermo. La vigilancia estrecha del drenaje resulta fundamental; en nuestro paciente el dolor se incrementó en las horas posteriores al procedimiento a pesar de una adecuada analgesia, pero también observando drenaje hemático. En cuanto al tratamiento, se inserta tubo de drenaje en aquéllos con aumento de volumen en radiografías seriadas, o en los moderados (mayores de $300 \mathrm{~mL}$ ) o masivos, conectándolos a un sistema valvular con sello de agua y aspiración continua a $20 \mathrm{cmH}_{2} \mathrm{O}$ (Pleur-evac). ${ }^{7,8}$

Por último, es importante destacar que, aunque el neumotórax espontáneo rara vez resulta mortal, éste puede ser compresivo, requieriendo descompresión inmediata con aguja, por riesgo de parada cardiorrespiratoria. $^{9}$

\section{REFERENCIAS}

1. Le Clainche L, Houdouin V. Neumotórax en el niño. EMC Pediatría. 2019; 54(2): 1-7.

2. Landete Rodríguez P, Acosta Gutiérrez CM, Hernández Olivo M, Milian Goicoechea H, Diab Cáceres L. Neumotórax. Medicine. 2018; 12(68): 3999-4005.

3. Casha AR, Manché A, Camilleri L, Gatt R, Dudek K, Pace-Bardon $\mathrm{M}$ et al. A biomechanical hypothesis for the pathophysiology of apical lung disease. Med Hypotheses. 2016; 92: 88-93.

4. Bintcliffe O, Maskell N. Spontaneous pneumothorax. BMJ. 2014; 348: g2928.

5. Aguinagalde B, Aranda JL, Busca P, Martínez I, Royo I, Zabaleta J; Grupo de trabajo de la GPC para el Manejo de Pacientes con Neumotórax espontáneo. SECT Clinical practice guideline on the management of patients with spontaneous pneumothorax. Cir Esp. 2018; 96(1): 3-11.

6. Williams K, Baumann L, Grabowski J, Lautz TB. Current practice in the management of spontaneous pneumothorax in children. $J$ Laparoendosc Adv Surg Tech A. 2019; 29(4): 551-556.

7. Cortes Telles A, Morales Villanueva CE, Figueroa Hurtado E. Hemotórax: etiología, diagnóstico, tratamiento y complicaciones. Rev Biomed. 2016; 27: 119-126.

8. González Fernández AM, Torres Torres AR, Valverde Molina J. Traumatismo torácico, neumotórax, hemoptisis y tromboembolismo pulmonar. Protoc Diagn Ter Pediatr. 2017; 1: 189-209.

9. Talbott MM, Campos A, Okorji O, Martel TJ. EMS pneumothorax identification without ancillary testing. 2020 Aug 30. In: StatPearls [Internet]. Treasure Island (FL): StatPearls Publishing; 2020. PMID: 30422463.

Conflicto de intereses: La autora declara que no tiene. 